\title{
HALF-MILLIMETER-RANGE VERTICALLY SCANNING MICROLENSES FOR MICROSCOPIC FOCUSING APPLICATIONS
}

\author{
Ankur Jain and Huikai Xie \\ Department of Electrical \& Computer Engineering, University of Florida \\ Gainesville, Florida 32611-6200, USA
}

\begin{abstract}
This paper presents the design, fabrication and operation of a new class of microlens scanners that can generate large vertical piston motion at low actuation voltages. These scanners are needed by endoscopic bio-imaging applications such as optical coherence microscopy (OCM) which require microlenses to axially scan their focal planes by 0.5 to $2 \mathrm{~mm}$. Photoresist reflow technique was used to form microlenses on lens holders that are integrated with largevertical-displacement (LVD) microactuators. The lens holders are fabricated using a modified post-CMOS micromachining process which can provide additional thermal isolation to the polymer microlens and form a transparent oxide mesh within the hollow lens-holders to enable formation of larger polymer microlenses. These scanners have demonstrated a maximum vertical displacement of $0.71 \mathrm{~mm}$ at an actuation voltage of $23 \mathrm{~V}$.
\end{abstract}

\section{INTRODUCTION}

Microlenses have been widely used for numerous opticalbeam focusing applications, which vary from optical communication to biomedical applications. Microlens scanners have been developed for lateral laser-beam scanning in optical switching systems [1], while vertically scanning microlenses have been used for biomedical imaging [2], and optical data storage applications [3]. Stationary microlenses with tunable focal lengths have been demonstrated for miniature cameras [4], microfluidic systems [5], and other microscopic focusing applications [6].

Another promising application for vertically-scanning microlenses is optical coherence microscopy (OCM), which is a biomedical imaging technology that combines the imaging capabilities of optical coherence tomography (OCT) and confocal microscopy to achieve high resolutions in both axial and transverse directions [7]. However, OCM achieves high transverse resolution at an expense of small imaging depth. In order to increase OCM's imaging depth, a lens scanner must be used to scan along the optical axis. OCM imaging requires vertical scanners that can provide an axial scanning depth of 0.5 to $2 \mathrm{~mm}$. The vertical scanning range of microlens scanners demonstrated by other research groups is far less than $0.1 \mathrm{~mm}$, and high driving voltages are often required $[2,3]$.

This paper presents microlens scanners that use large-verticaldisplacement (LVD) microactuators to generate half-millimeterrange vertical scans at low actuation voltages; and these devices are also small enough to be packaged inside slender catheters for endoscopic OCM imaging. At Hilton Head 2004, the LVD microactuation mechanism was introduced for the first time [8], and then a prototype LVD microlens scanner with a vertical scan range of $0.28 \mathrm{~mm}$ was presented at MEMS 2005 [9]. In this paper, we report new LVD microlens scanners with vertical displacements up to $0.71 \mathrm{~mm}$ at $23 \mathrm{~V}$. These newly designed scanners provide better thermal isolation for the polymer microlens using two different approaches. Another innovation is the addition of a transparent oxide mesh within the hollow lens-holder to enable formation of larger polymer microlenses.

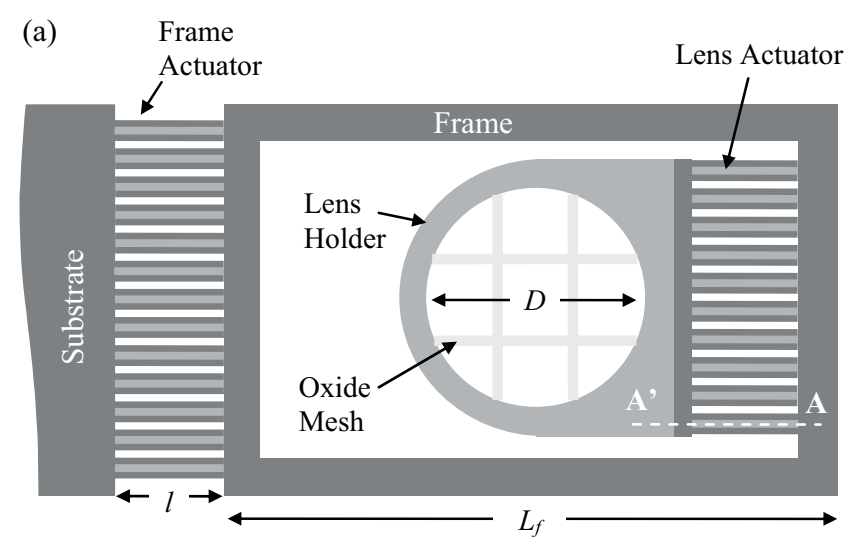

(b)

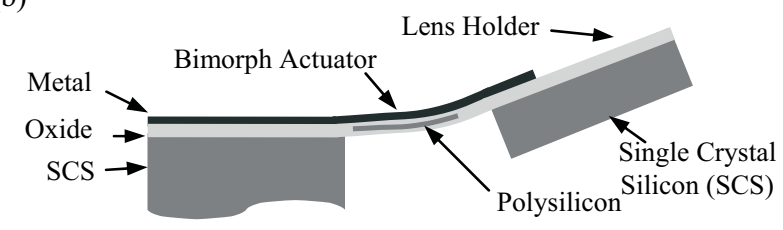

Figure 1. (a) Top-view design schematic of the LVD scanner. Actuator length, $l=200 \mu \mathrm{m}$; Frame length, $L_{f}=1.6 \mathrm{~mm}$; Lensopening diameter, $D=0.6 \mathrm{~mm}$. (b) Cross-sectional view of $A-A$ '.

\section{SCANNER DESIGN \& FABRICATION}

The structure of this scanner consists of a polymer microlens integrated on a MEMS lens-holder, which is thermally-actuated using the LVD mechanism. The schematic drawing of the LVD scanner is illustrated in Fig. 1, where the lens-holder is attached to a rigid silicon frame by a set of aluminum/silicon-dioxide bimorph beams (lens actuator). A $0.6-\mathrm{mm}$ diameter circular opening in the center of the lens-holder allows light to pass through, and it also contains a transparent silicon-dioxide mesh structure. This mesh structure is designed to hold the polymer microlens, and it also prevents smaller polymer droplets from falling through during the microlens formation process step. The structural rigidity of the lens-holder plate and the frame are maintained by a 30 to $40-\mu \mathrm{m}$ thick single-crystal-silicon (SCS) layer. The 1.6-mm long frame is attached to the substrate by another set of identical bimorph beams (frame actuator). These actuators together form an LVD microactuator set, in which the curls of the two sets of bimorph beams compensate each other resulting in zero initial tilt of the lens-holder. The polysilicon resistors embedded in the bimorph beams are used for electrothermal actuation. The LVD microactuator provides large piston motion by simultaneously applying voltage to both actuators, so that equal angular rotation by both actuators results in pure vertical displacement of the microlens. Further details about the LVD actuation principle have been previously reported in [8-10]. 
(a)

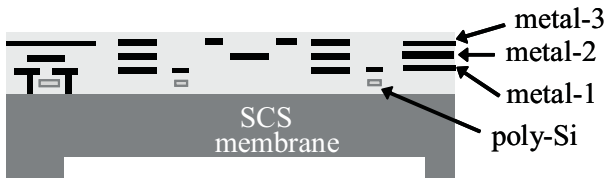

(b)

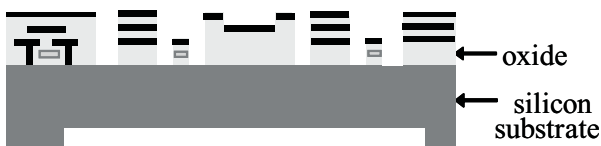

(c)

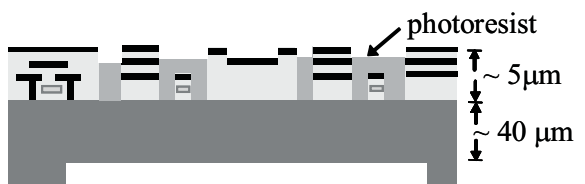

(d)

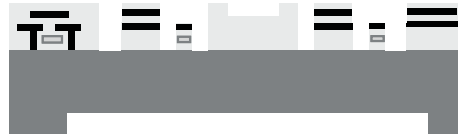

(e)

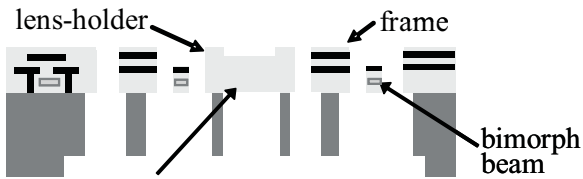

oxide mesh

(f)

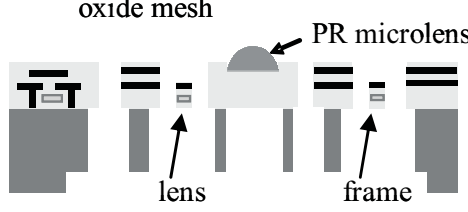

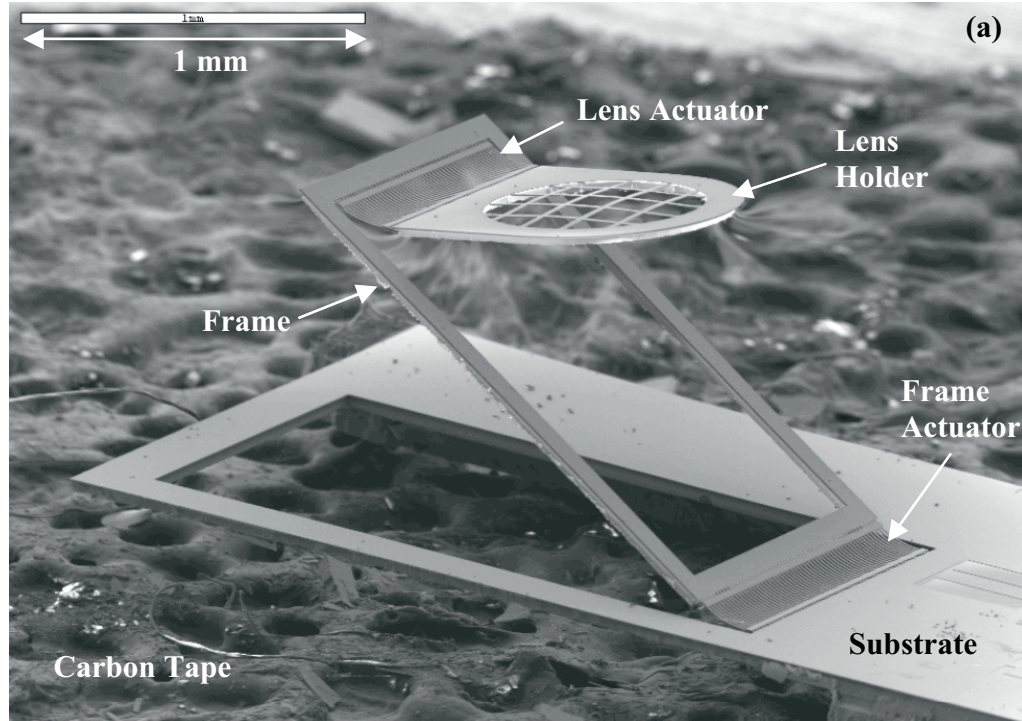
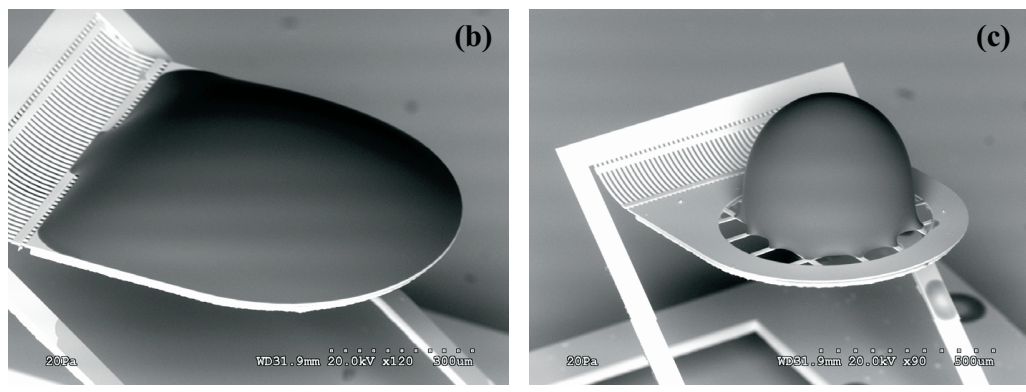

Figure 2. Modified CMOS-MEMS fabrication process. (a) Backside Si etch. (b) Frontside oxide etch. (c) PR spun-on and etched to protect Metal-1. (d) Wet etch of exposed layers of Metal's 2 \& 3. (e) Deep Si trench etch, followed by Si undercut. (f) Microlens formation.

Figure 3. SEM images of: (a) Fabricated device before microlens formation (b) $3 \mathrm{~mm}$ focal length convex PR microlens formed on entire lens holder plate. (c) $0.5 \mathrm{~mm}$ focal length PR ball-lens formed on the mesh area only. These PR lenses were imaged using a variable-pressure environmental SEM.

In order to form a transparent oxide mesh within the $600-\mu \mathrm{m}$ diameter opening of the lens-holder, the post-CMOS-MEMS fabrication process used in [9] was modified to include two additional etch steps, as illustrated in Fig. 2. After the backside Si and frontside oxide etch steps [Figs. 2(a) and 2(b)], a planar photoresist (PR) layer is spun on and dry-etched to expose the metal-3 and metal-2 layers only ${ }^{1}$ [Fig. 2(c)]. Next, the exposed metal layers are completely removed using a wet aluminum etchant, and the remaining PR layer is stripped off [Fig. 2(d)]. The device is then released using a deep Si trench etch, followed by an isotropic Si undercut to etch the SCS from beneath the bimorph beams to form the thin-film actuators [Fig. 2(e)]. Finally, PR droplets are precisely dispensed on the lens-holder to form the polymer microlens due to surface tension [Fig. 2(f)]. The process modifications allow the use of the metal-2 layer to define the structure for the 3- $\mu \mathrm{m}$ thick oxide mesh within the lens holder. Also, the PR microlens is now formed on a layer of insulating oxide instead of a more thermally-conductive Al metal layer, resulting in better thermal isolation from the electrically-heated lens-actuator. Thermally isolating PR microlenses from heating sources prevents the situations in [11], where the focal length of a polymer microlens changed significantly with increasing heat. Thermal isolation also prevents carbonization of the PR microlens, which tends to occur at temperatures greater than $200^{\circ} \mathrm{C}[\mathbf{1 2}]$.

\footnotetext{
${ }^{1}$ For die level fabrication, lithographic techniques can be used to expose the metal-2 and metal-3 layers on the lens-holder only.
}

The initial rest position of a fabricated microlens [Fig. 3(a)] is $1.2 \mathrm{~mm}$ above the substrate surface. In order to form a polymer microlens on the elevated lens-holder, droplets of PR are first dispensed using a nanoliter-injection system and then baked in an oven to form a microlens due to surface tension [12]. Focal length can be controlled by varying the quantity and/or volume of the dispensed dropets. PR microlenses with focal lengths between 0.5 to $3 \mathrm{~mm}$ with numerical apertures (NA) ranging from 0.1 to 0.35 have been successfully fabricated. Figs. 3(b) and 3(c) show two fabricated PR microlenses with different focal lengths and lens sizes. To test the imaging quality of these microlenses, a chrome
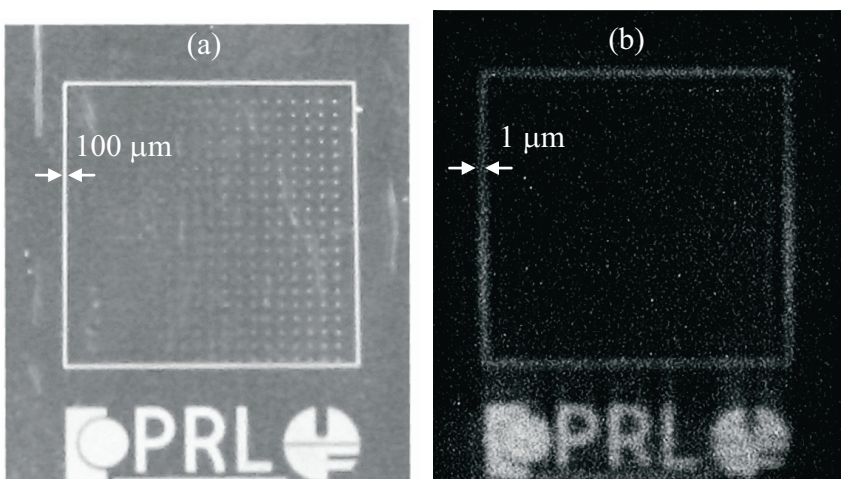

Figure 4. Imaging using the PR microlens. (a) Photo of the test pattern on a chrome mask. (b) Corresponding image of the test pattern as seen at the focal plane of the PR microlens. 


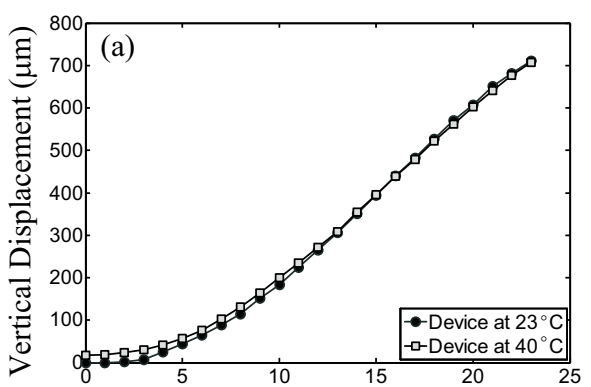

Frame Actuator Voltage (V)
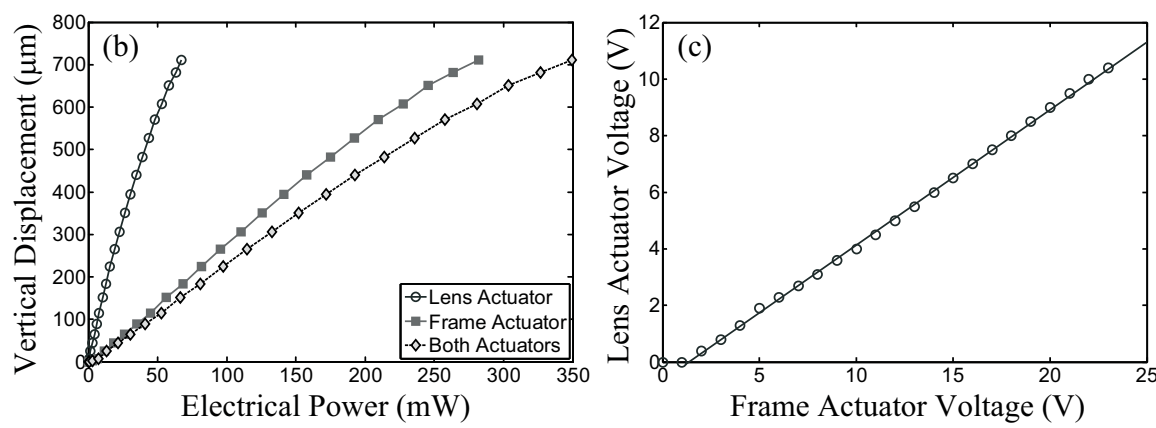

Figure 5. Plots of the vertical displacement of the microlens as a function of: (a) frame actuator voltage, and (b) applied electrical power. (c) Corresponding plot of the actuation voltages applied to the two actuators to obtain the vertical displacement in (a) and (b).

test mask was placed $\sim 100 \mathrm{~mm}$ away from the ball-type microlens, and the image at its focal plane (which is de-magnified by about $100 \times$ ) was captured using a CCD microscope camera. The test pattern image obtained by a PR microlens with a numerical aperture of 0.35 is shown in Fig. 4, where features as small as $1 \mu \mathrm{m}$ can be resolved.

\section{EXPERIMENTAL RESULTS}

Vertical displacement of the PR microlens was achieved by simultaneously exciting both actuators and tuning the actuation voltages such that the opposite angular rotations of the two actuators offset any net tilting of the microlens. The static displacements of the microlens and its corresponding driving voltage plots are shown in Fig. 5, where the vertical displacements of the microlens were observed to within an accuracy of $\pm 2 \mu \mathrm{m}$ by using a $40 \times$ objective lens $(0.65$ NA). Fig. 5(a) shows that a maximum vertical displacement of $0.71 \mathrm{~mm}$ was obtained at a dc voltage of $23 \mathrm{~V}$ applied to the frame actuator. Fig. 5(b) displays the same vertical displacement data, but with respect to the electrical power supplied to the two actuators. The plot of the two driving voltages that are required to obtain this vertical displacement is shown in Fig. 5(c). The slope of this linear plot yields the driving voltage ratio for the two actuators for LVD actuation. The driving voltage applied to the lens actuator is $43 \%$ of the frame actuator voltage, which is provided by a voltage divider. Using this constant voltage ratio, the maximum tilt of the microlens in the entire $0.71 \mathrm{~mm}$ travel range is less than $0.4^{\circ}$.

There is significant lateral shifting of the microlens during vertical actuation, as shown in Fig. 6(a). A maximum lateral shift of $425 \mu \mathrm{m}$ is observed for the entire vertical scan range. This lateral shift is mainly due to the rotational displacement of the frame actuator, and is amplified by the long length of the silicon frame. This lateral shift will be accounted for and corrected during the OCM image formation stage.
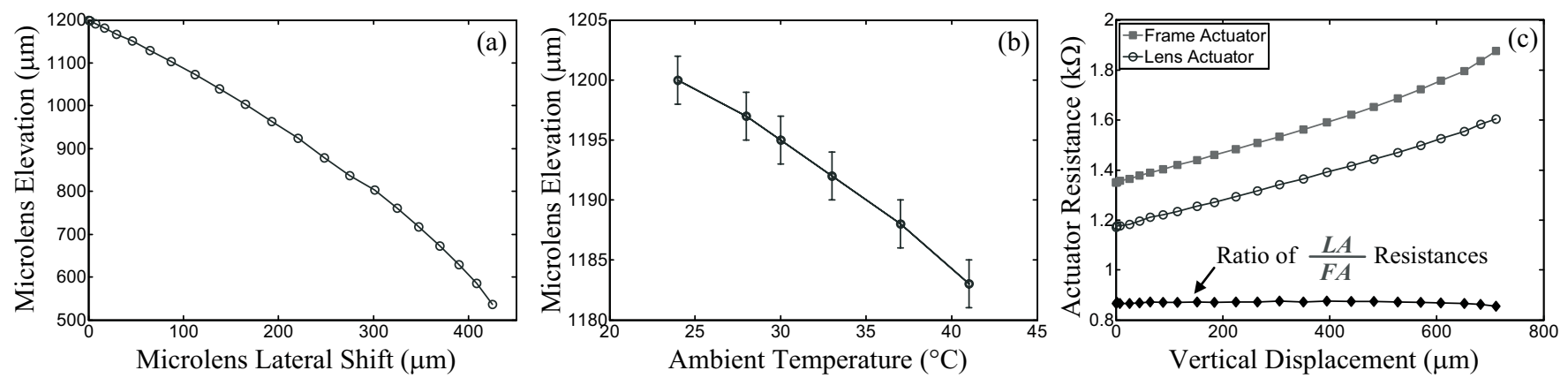

Figure 6. (a) Plot of the observed lateral shift of the microlens during LVD actuation. (b) Initial elevation of the microlens as a function of the ambient temperature. (c) Plot showing the linear increase in polysilicon heater resistance of the two actuators during LVD actuation of the microlens.
The initial elevations of the microlens at different operating temperatures were also documented, and the results are presented in Fig. 6(b). The initial lens-holder elevation of a fabricated microlens is $1.2 \mathrm{~mm}$ at a room temperature $\left(23^{\circ} \mathrm{C}\right)$, and it decreases with increasing temperature. Since this device will be packaged inside an endoscope for in vivo OCM imaging, the maximum ambient temperature shall not exceed $40^{\circ} \mathrm{C}$. Therefore, the vertical scan range of the device will, in the worst case, be reduced by up to $20 \mu \mathrm{m}$, which is less than $3 \%$ of the entire scan range. This can be seen in the plots of Fig. 5(a) where an increase in ambient temperature to $40^{\circ} \mathrm{C}$ reduced the vertical scan range by $\sim 17 \mu \mathrm{m}$.

The resistances of the polysilicon heaters embedded in both actuators increase significantly with applied voltage due to Joule heating. Joule heating raises the temperature of the bimorphs, which in turn increases the heater resistances due to the thermal coefficient of resistivity of polysilicon. The open-circuit, roomtemperature electrical resistances of the lens and frame actuators are $1.17 \mathrm{k} \Omega$ and $1.35 \mathrm{k} \Omega$, respectively. A linear correlation between the vertical displacement of the microlens and the heater resistance of the two actuators was observed, as shown in Fig. 6(c). This experimentally determined linear relationship between the resistors allows for closed-loop feedback control of the vertical position of the microlens by monitoring the polysilicon resistance change of each actuator.

\section{MICROLENS SCANNER DESIGN 2}

Another LVD microlens scanner (design illustrated in Fig. 7(a)) that uses thin-film isolation beams to thermally isolate the PR microlens was fabricated using the unmodified CMOSMEMS process used in [9]. This design also provides thermal conduction metal lines that preferentially conduct heat away from the two frames and towards the substrate. The SEM of a fabricated device with the lens-holder elevated $0.5 \mathrm{~mm}$ above the substrate 

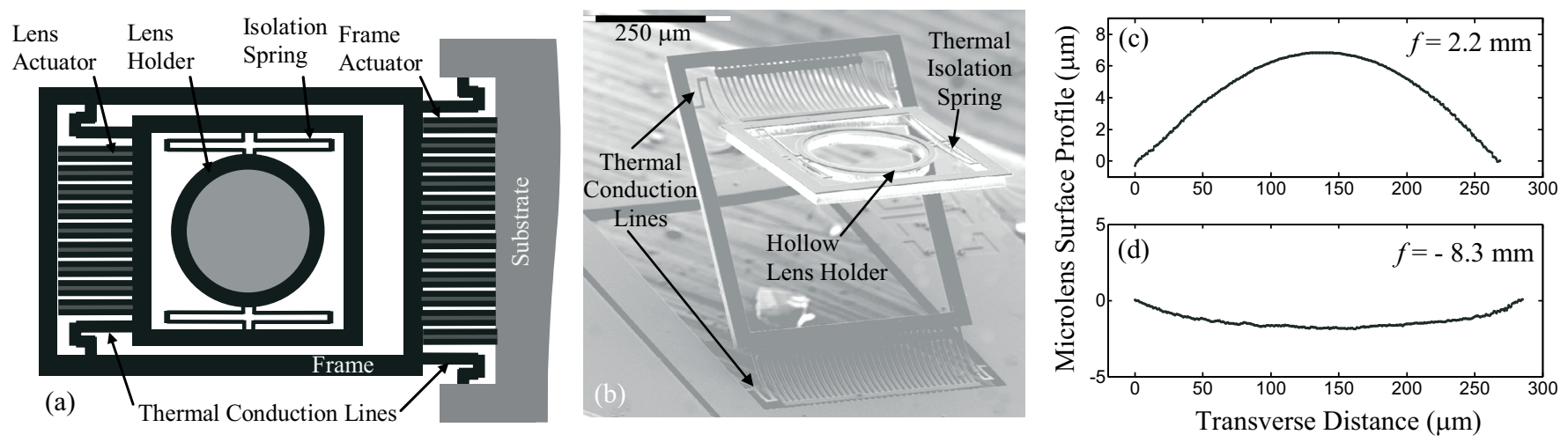

Figure 7. Microlens Scanner Design 2. (a) Schematic showing the thermal isolation spring and thermal conduction lines. (b) SEM of a fabricated scanner showing that the initial elevation of the lens holder is $0.5 \mathrm{~mm}$ above the substrate plane. Surface profiles of the (c) convex and (d) concave PR microlenses formed within the lens holder of Design 2.

plane is shown in Fig. 7(b). Microlenses were formed by dispensing a PR droplet into the hollow 280- $\mu \mathrm{m}$ diameter lensholder. Depending on the volume of the PR droplet dispensed, it is possible to obtain convex or concave microlenses. The profiles of convex and concave microlenses, as obtained using a white-light optical profilometer, are shown in Figs. 7(c) and 7(d). The maximum microlens displacement is $0.32 \mathrm{~mm}$ at a frame actuator voltage of $21 \mathrm{~V}$, as shown in Fig. 8. The same voltage divider was used to drive this device.

The increase in thermal isolation to the PR microlens due to the isolation springs, and the thermal effectiveness of adding thermal conduction lines are still under investigation.

\section{CONCLUSIONS}

Half-millimeter-range vertically scanning microlenses have been successfully demonstrated. The CMOS-MEMS fabrication process was modified in order to allow the formation of a transparent silicon-dioxide mesh within the hollow microlens holder. This mesh allowed the formation of larger PR microlenses, and it also provided additional thermal isolation to the polymer lenses from the electrically-heated LVD actuators. A fabricated microlens scanner demonstrated a maximum vertical displacement of $0.71 \mathrm{~mm}$ at an actuation voltage of $23 \mathrm{~V}$. The large actuation range and small device footprint make this LVD microlens scanner very suitable for use in endoscopic OCM and other microscopic focusing applications.

\section{ACKNOWLEDGEMENTS}

The authors would like to thank T. Riedhammer for the VP-SEM images. This project is supported by the National Science Foundation Biophotonics Program under award BES-0423557.

\section{REFERENCES}

[1]. H. Toshiyoshi, G. -D. J. Su, J. LaCosse, and M. C. Wu, "A surface micromachined optical scanner array using photoresist lenses fabricated by a thermal reflow process," Journal of Lightwave Technology 21, pp. 1700-1708 (2003).

[2]. S. Kwon, V. Milanovic, and L. P. Lee, "Vertical microlens scanner for 3D imaging," 2002 Solid State Sensor and Actuator Workshop, Hilton Head, SC, June 2002, pp. 227230.

[3]. S.-H. Kim, Y. Yee, J. Choi, H. Kwon, M.-H. Ha, C. Oh, and J. U. Bu, "Integrated micro optical flying head with lens

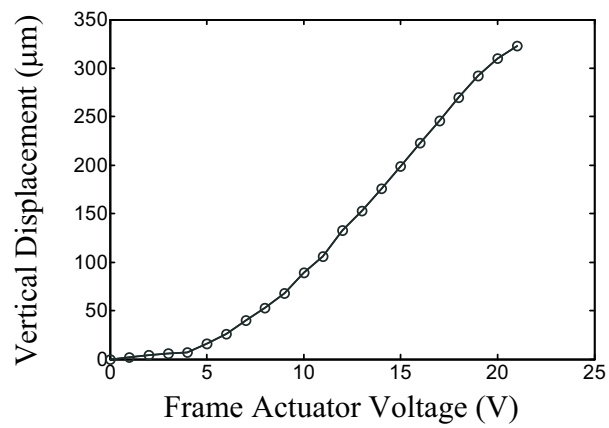

Figure 8. Vertical displacement of the microlens of Design 2.

positioning actuator for small form factor data storage," Transducers 2003, Boston, MA, June 2003, pp. 607-610.

[4]. S. Kuiper and B. H. W. Hendriks, "Variable-focus liquid lens for miniature cameras," Applied Physics Letters 85, pp. 11281130 (2004).

[5]. N. Chronis, G. L. Liu, K. -H. Jeong, and L. P. Lee, “Tunable liquid-filled microlens array integrated with microfluidic network," Optics Express 11, pp. 2370-2378, (2003).

[6]. W. Wang, J. Fang, and K. Varahramyan, "Compact variablefocusing microlens with integrated thermal actuator and sensor," IEEE Photonics Technology Letters 17, pp. 26432645 (2005).

[7]. A. D. Aguirre, P. Hsiung, T. H. Ko, I. Hartl, and J. G. Fujimoto, "High-resolution optical coherence microscopy for high-speed, in vivo cellular imaging," Optics Letters 28, pp. 2064-2066, (2003).

[8]. A. Jain, H. Qu, S. Todd, G. K. Fedder, and H. Xie, "Electrothermal SCS micromirror with large-verticaldisplacement actuation," 2004 Solid State Sensor, Actuator and Microsystems Workshop, Hilton Head, SC, June 2004, pp. 228-231.

[9]. A. Jain and H. Xie, "A tunable microlens scanner with largevertical-displacement actuation," IEEE MEMS 2005, Miami Beach, FL, January 2005, pp. 92-95.

[10].A. Jain, S. Todd, and H. Xie, "An electrothermally-actuated, dual-mode micromirror for large bi-directional scanning," IEDM 2004, San Francisco, CA, December 2004, pp. 47-50.

[11].A. L. Glebov, L. Huang, S. Aoki, M. Lee, and K. Yokouchi, "Planar hybrid polymer-silica microlenses with tunable beamwidth and focal length," IEEE Photonics Technology Letters 16, pp. 1107-1109 (2004).

[12].D. Daly, Microlens Arrays, Taylor \& Francis: London, 2001. 